\title{
Transition Radiation Wakefields for a Beam Passing through a Metallic Foil*
}

\author{
Karl L.F. Bane and Gennady Stupakov \\ Stanford Linear Accelerator Center, \\ Stanford University, Stanford, CA 94309
}

\begin{abstract}
A proposal for the LCLS has been made to generate a shorter light pulse by placing a spoiler foil in the middle of a compressor chicane: The foil has a small slot, which selects out the small fraction of particles passing through it ("target particles") to lase [1],[2]. In this report, using the method of field matching, we obtain longitudinal and transverse impedances and wakefields for several models of the proposed LCLS spoiler foil. Our models have no slot. We consider the model of a pencil beam, and of a cylindrically symmetric, bi-Gaussian beam that is wider than it is long. Thirdly, we generate a Green function that allows us to consider asymmetric beams also. For target particles of the tilted, tri-Gaussian beam that is found at the LCLS spoiler location we obtain approximate analytical formulas and numerical results for wakefield kicks in the three directions. We find that the kicks, after correction using a simple dipole and and quadrupole, are all within tolerances.
\end{abstract}

*Work supported by the Department of Energy, contract DE-AC03-76SF00515 


\section{INTRODUCTION}

The Linac Coherent Light Source (LCLS) [3] will produce SASE radiation in the angstrom wavelength range. One configuration of this project envisions having only a few femtosecond portion of the 200 femtosecond long electron bunch lasing. The idea for preparing the beam to lase in this way is to have it pass through a thin foil "spoiler" with a small slot in the middle of the second chicane compressor (see Fig. 1) [1],[2]. Most of the beam electrons pass through the foil, and their emittance is increased due to scattering in the foil. A small fraction of the beam passes through the slot, and the emittance of these electrons should remain unperturbed.

A rough sketch of the beam and foil is given in Fig. 1. A thin, round conducting foil, with a narrow slot of half-width $\Delta x$, is positioned within a cylindrically-symmetric, metallic beam pipe of radius $a$. An ultra-relativistic beam passes centered through the beam pipe. The beam charge distributions are Gaussian in the three directions, with rms beam sizes $\sigma_{x}, \sigma_{z}$, and $\sigma_{y}$. The beam passes with an $x-z$ correlation, described by angle $\alpha$ with respect to the $x$ axis; the rms "slice length" - the length in $z$ for a given $x$-is $\sigma_{z s}$. Suggested parameters that will be used in estimations below are given in Table I. Note that $a \gg \sigma_{x} \gg \sigma_{z}, \sigma_{y} \gg \sigma_{z s}$; that $\alpha \approx \sqrt{\sigma_{z}^{2}-\sigma_{z s}^{2}} / \sigma_{x} \approx \sigma_{z} / \sigma_{x}$; and that the slice rms length is small, $\sigma_{z s} \approx \sqrt{\sigma_{z}^{2}-\alpha^{2} \sigma_{x}^{2}}$.

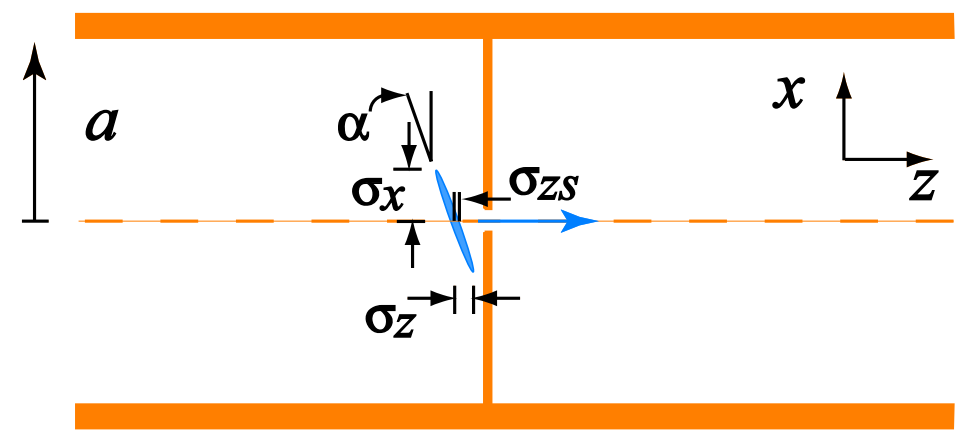

FIG. 1: A sketch in the $x-z$ plane of the beam and the foil with its slot. The beam pipe is round but the slot is flat, with half-width $\Delta x$. The beam is tilted in $x$ - $z$ by angle $\alpha(\ll 1)$, with the longitudinal slice rms length, $\sigma_{z s}$. Note that $a \gg \sigma_{x} \gg \sigma_{z}, \sigma_{y} \gg \sigma_{z s}$.

We are interested in the wakefield acting on the particles passing through the slot, i.e. that part of the beam with $|x| \leq \Delta x$ and whose size in $y$, slice size in $z$, and $x$ - $z$ correlation 
TABLE I: Parameters at the LCLS spoiler.

\begin{tabular}{lccl}
\hline \hline Beam Energy & $E$ & 4.5 & $\mathrm{GeV}$ \\
Bunch Charge & $e N$ & 1 & $\mathrm{nC}$ \\
Horizontal Bunch Divergence & $\sigma_{x^{\prime}}$ & 2 & $\mu \mathrm{rad}$ \\
Vertical Bunch Divergence & $\sigma_{y^{\prime}}$ & 2 & $\mu \mathrm{rad}$ \\
Intrinsic Energy Spread & $\left(\sigma_{\delta}\right)_{i n}$ & 6 & $10^{-5}$ \\
Horizontal Beam Size & $\sigma_{x}$ & 2.6 & $\mathrm{~mm}$ \\
Vertical Beam Size & $\sigma_{y}$ & 0.1 & $\mathrm{~mm}$ \\
Bunch Length & $\sigma_{z}$ & 0.1 & $\mathrm{~mm}$ \\
Slice Bunch Length & $\sigma_{z s}$ & 2 & $\mu \mathrm{m}$ \\
Beam Tilt Angle & $\alpha$ & 40 & $\mathrm{mrad}$ \\
Radius of Beam Pipe & $a$ & 20 & $\mathrm{~mm}$ \\
Spoiler Slot Half-Width & $\Delta x$ & 0.125 & $\mathrm{~mm}$ \\
\hline \hline
\end{tabular}

angle are characterized by $\sigma_{y}, \sigma_{z s}, \alpha$, respectively (we will call these "the target beam particles"). Since the width of the slot is much smaller than $\sigma_{x}$, only a small fraction of beam particles passes through the slot. We believe that, to good approximation, we can neglect the effect of the slot, and, in this report, we calculate the longitudinal and transverse wakes for a foil without slot. Note that an important feature of this problem is that the beam pipe radius $a$ is much larger than any beam dimension, a fact that will allow us to obtain results of rather simple analytical form. Note also that the model problem that we are about to solve - the wakefields of a foil without slot — gives, in fact, the wakefields associated with the transition radiation of a beam passing through a foil.

The organization of the paper is as follows: We begin in Section II by calculating the longitudinal impedance and wakefield of a pencil beam (one with zero transverse size). In Section III we consider a beam that is wider than long, but still cylindrically-symmetric, which is a more appropriate model for the LCLS spoiler problem, and obtain first the longitudinal and then the transverse wakes due to monopole modes. In Section IV we improve our model and generate a Green function for the transverse wake, from which 
we obtain the wakes of a tilted, tri-Gaussian beam. Finally, we apply the results to the LCLS spoiler parameters, to estimate the effect on the target particles of the LCLS beam. Appendix A gives the solution of an auxiliary sum used in Section II, and Appendix B presents more details of the calculation for the longitudinal wake in Section III.

\section{PENCIL BEAM}

\section{A Impedance Calculation}

Consider the geometry sketched in Fig. 2. It consists of a cylindrically symmetric beam pipe of radius $a$, with a closed, metallic foil at longitudinal position $z=0$. The pipe and foil are assumed to be perfectly conducting. An exciting particle of charge $e N$ moves on axis at the speed of light, from $z=-\infty$ to $\infty$; it passes through the foil at time $t=0$. An ultra-relativistic test particle follows behind at distance $s$ and at offset $r$ from the axis. We denote the two regions within the pipe as Region I $(z<0)$ and Region II $(z>0)$.

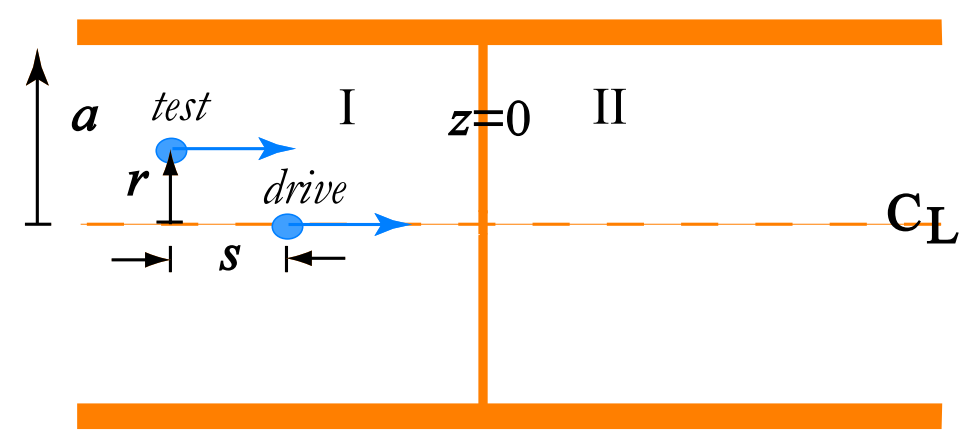

FIG. 2: A sketch of the geometry of our cylindrically-symmetric model showing the two regions, I and II, and the relative positions of the drive and test particles.

We follow a standard method to find the wakefield, which is to first obtain the impedance, and then to perform a Fourier transform. The longitudinal wakefield - the total voltage loss 
of the test particle in its traversal from $z=-\infty$ to $\infty$, divided by $e N$-is given by

$$
\begin{aligned}
W_{z}(r, s) & =-\left.\frac{1}{e N} \int_{-\infty}^{\infty} E_{z}(r, z ; t) d z\right|_{t=(s+z) / c} \\
& =-\frac{1}{e N} \int_{-\infty}^{\infty} d z \int_{-\infty}^{\infty} d t \int_{-\infty}^{\infty} \frac{d \omega}{2 \pi} E_{z}(r, z ; t) e^{i \omega[t-(s+z) / c]} \\
& =\frac{1}{2 \pi} \int_{-\infty}^{\infty} d \omega e^{-i \omega s / c}\left[-\frac{1}{e N} \int_{-\infty}^{\infty} d z \tilde{E}_{z}(r, z ; \omega) e^{-i \omega z / c}\right],
\end{aligned}
$$

where $E_{z}$ is the electric field in the longitudinal direction, and the tilde denotes the Fourier transform of a quantity, which we define by

$$
\tilde{A}(\omega)=\int_{-\infty}^{\infty} A(t) e^{i \omega t} d t .
$$

The quantity in brackets in the last line of Eq. 1 is the longitudinal impedance:

$$
Z(\omega)=-\frac{1}{e N} \int_{-\infty}^{\infty} \tilde{E}_{z}(r, z ; \omega) e^{-i \omega z / c} d z
$$

Note that it is just the Fourier transform of the wake.

In the problem of Fig. 2, the driving particle excites TM modes in both Regions I and II. The non-zero components of the (Fourier transform of the) excited fields can be written as infinite sums:

$$
\begin{aligned}
& \tilde{E}_{z}= \pm \frac{1}{a^{2}} \sum_{n} C_{n} j_{0 n}^{2} J_{0}\left(\frac{j_{0 n} r}{a}\right) e^{ \pm i k_{z n} z} \\
& \tilde{E}_{r}=-\frac{i}{a} \sum_{n} C_{n} k_{z n} j_{0 n} J_{1}\left(\frac{j_{0 n} r}{a}\right) e^{ \pm i k_{z n} z} \\
& \tilde{H}_{\phi}= \pm \frac{i \omega}{c a} \sum_{n} C_{n} j_{0 n} J_{1}\left(\frac{j_{0 n} r}{a}\right) e^{ \pm i k_{z n} z}
\end{aligned}
$$

where the longitudinal propagation constant is given by

$$
k_{z n}=\sqrt{\left(\frac{\omega}{c}\right)^{2}-\left(\frac{j_{0 n}}{a}\right)^{2}} ;
$$

with $J_{n}$ the Bessel function of the first kind of order $n$ and $j_{0 n}$ the $n^{\text {th }}$ zero of $J_{0}(x)$. Note that there is a symmetry between the fields in the two regions excited by particle, and in Eqs. 4-6 the upper symbols indicate fields in Region I, the lower symbols those in Region II.

The Coulomb source field of the ultra-relativistic driving particle is given by

$$
\left(E_{r}\right)_{s}=\frac{2 e N}{r} \delta(z-c t) .
$$


When the source particle impinges on the conducting wall, it excites radiation fields that cancel the source field at $z=0$, since the total $E_{r}$ must equal zero on the foil. We obtain the radiation field expansion coefficients $C_{n}$ by field matching at $z=0$ :

$$
\tilde{E}_{r}(z=0)=-\frac{i}{a} \sum_{n^{\prime}} C_{n^{\prime}} k_{z n^{\prime}} j_{0 n^{\prime}} J_{1}\left(\frac{j_{0 n^{\prime}} r}{a}\right)=-\frac{2 e N}{c r} .
$$

Multiplying both sides by $J_{1}\left(j_{0 n} r / a\right) r d r$ and then integrating from 0 to $a$ we obtain

$$
C_{n}=-\frac{4 i e N}{c k_{z n} j_{0 n}^{2} J_{1}^{2}\left(j_{0 n}\right)} \text {. }
$$

Now we can obtain the longitudinal impedance using Eq. 3. Let us, for the moment, limit ourselves to the case $r=0$ (a test particle on axis). We find that

$$
\begin{aligned}
Z(\omega) & =-\frac{1}{e N a^{2}} \sum_{n} C_{n} j_{0 n}^{2}\left[\int_{-\infty}^{0} e^{i z\left(k_{z n}-\omega / c\right)} d z-\int_{0}^{\infty} e^{i z\left(-k_{z n}-\omega / c\right)} d z\right] \\
& =\frac{8}{c} \sum_{n} \frac{1}{\sqrt{1-\left(\frac{j_{0 n} c}{\omega a}\right)^{2}} j_{0 n}^{2} J_{1}^{2}\left(j_{0 n}\right)} .
\end{aligned}
$$

The real part of the impedance $R(\omega)$ is given by the same formula, but with $\sum_{n}$ replaced by $\sum_{n}^{\prime}$, which means to sum over all $n$ for which $k_{z n}$ is real (propagating modes), i.e. for all $n$ with $j_{0 n} \leq \omega a / c$. Note that $R(\omega)$ can also be obtained from the energy balance equation $R(\omega)=\pi /(e N)^{2}(d U / d \omega)$, where $d U / d \omega$ is the energy radiated, and given by (see, e.g. Ref. [4])

$$
\frac{d U}{d \omega}=\frac{\omega}{2 \pi} \sum_{n}^{\prime} k_{z n} j_{0 n}^{2} J_{1}^{2}\left(j_{0 n}\right)\left|C_{n}\right|^{2} .
$$

(Note that half the energy is radiated in Region I and half in Region II.) We find that the real part of the impedance is again given by

$$
R(\omega)=\frac{8}{c} \sum_{n}^{\prime} \frac{1}{\sqrt{1-\left(\frac{j_{0 n} c}{\omega a}\right)^{2}} j_{0 n}^{2} J_{1}^{2}\left(j_{0 n}\right)} .
$$

\section{B High frequency solution}

In the case of large $\omega a / c$ we can perform the sum of Eq. 14. We first rewrite

$$
\begin{aligned}
\sum_{n}^{\prime} \frac{1}{\sqrt{1-\left(\frac{j_{0 n} c}{\omega a}\right)^{2}} j_{0 n}^{2} J_{1}^{2}\left(j_{0 n}\right)} & =\sum_{n}^{\prime}\left[\frac{1}{\sqrt{1-\left(\frac{j_{0 n} c}{\omega a}\right)^{2}} j_{0 n}^{2} J_{1}^{2}\left(j_{0 n}\right)}-\frac{1}{j_{0 n}^{2} J_{1}^{2}\left(j_{0 n}\right)}\right] \\
& +\sum_{n}^{\prime}\left[\frac{1}{j_{0 n}^{2} J_{1}^{2}\left(j_{0 n}\right)}-\frac{1}{2 n}\right]+\sum_{n}^{\prime} \frac{1}{2 n} .
\end{aligned}
$$


Consider the 3 pieces of sum $S=S_{1}+S_{2}+S_{3}$. The first sum $S_{1}$ has its largest contributions at large $n$. Therefore, using large $n$ approximations $j_{0 n} \approx n \pi$ and $J_{1}\left(j_{0 n}\right) \approx \sqrt{2 / n}(-1)^{n} / \pi$, and replacing the sum with an integral, we obtain

$$
S_{1} \approx \frac{1}{2} \int_{0}^{1} \frac{d x}{x}\left(\frac{1}{\sqrt{1-x^{2}}}-1\right)=\frac{1}{2} \ln 2 .
$$

The dominant contribution to $S_{2}$ is for low $n$; the result is $S_{2}=\frac{1}{2} \ln (\pi / 2)$ (see the Appendix A). Since $\sum_{n}^{\prime}$ means to $\operatorname{sum}$ up to $n \approx \omega a /(\pi c), S_{3}=\frac{1}{2}\left[\ln (\omega a /(\pi c))+\gamma_{E}\right]$, where $\gamma_{E} \approx 0.577$, the Euler constant. Finally, we obtain

$$
R(\omega)=\frac{4}{c}\left(\gamma_{E}+\ln \frac{\omega a}{c}\right)
$$

Note that if $c /(a \omega)$ is small compared to $1 / \gamma$, with $\gamma$ the Lorentz energy factor, then the quantity $(\omega a / c)$ in Eq. 17 should be replaced by $\gamma$. This is the result that obtains when $a \rightarrow \infty$, and it is this form of the equation (except for the $\gamma_{E}$ term) that corresponds to the transition radiation $d U / d \omega$ found in the literature [5].

\section{Loss factor}

The loss factor of a bunch - the total voltage lost by the bunch divided by its charge - is given by

$$
\kappa_{t o t}=\frac{1}{\pi} \int_{0}^{\infty} R(\omega)\left|\tilde{\lambda}_{z}(\omega)\right|^{2} d \omega,
$$

with $\lambda_{z}(s)$ the longitudinal bunch distribution. For a Gaussian bunch of rms length $\sigma_{z}$, $\lambda_{z}(s)=1 /\left(\sqrt{2 \pi} \sigma_{z}\right) e^{-\frac{1}{2} s^{2} / \sigma_{z}^{2}}$ and $\tilde{\lambda}_{z}(\omega)=e^{-\frac{1}{2} \omega^{2} \sigma_{z}^{2} / c^{2}} ;$ therefore,

$$
\kappa_{t o t}=\frac{1}{\sqrt{\pi} \sigma_{z}}\left(\gamma_{E}+2 \ln \frac{a}{2 \sigma_{z}}\right) \text {. }
$$

We have used the relation

$$
\int_{0}^{\infty} e^{-x^{2}} \ln x d x=-\frac{\sqrt{\pi}}{4}\left(\gamma_{E}+2 \ln 2\right) .
$$




\section{Longitudinal Wakefield}

The wakefield can be obtained from $R(\omega)$ using

$$
W(s)=\frac{2}{\pi} H(s) \int_{0}^{\infty} R(\omega) \cos \frac{\omega s}{c} d \omega
$$

where $H(s)=1$ for $s>0,0$ for $s<0$. In our case the wakefield turns out to be rather singular, and a more convenient response function is the step function response $S(s)$, the integral of the wake:

$$
\begin{aligned}
S(s) & \equiv \int_{0}^{s} W\left(s^{\prime}\right) d s^{\prime} \\
& =\frac{2 c}{\pi} H(s) \int_{0}^{\infty} \frac{R(\omega)}{\omega} \sin \frac{\omega s}{c} d \omega .
\end{aligned}
$$

Using the relation

$$
\int_{0}^{\infty} \frac{\ln x}{x} \sin x y d x=-\frac{\pi}{2}\left(\gamma_{E}+\ln y\right) \operatorname{Sign}(y)
$$

we finally obtain

$$
S(s)=4 H(s) \ln \frac{a}{s} .
$$

Note that our result is similar to the step function response for a beam passing between two parallel plates, but where the cut-off $a$ is replaced by twice the distance between the plates $[6]$.

\section{E Bunch Wake}

The bunch wake is the voltage induced by a bunch divided by its charge. For a bunch with longitudinal distribution $\lambda_{z}(s)$ [assuming $\lambda_{z}(\infty)=0$ ] the bunch wake is given by

$$
\mathcal{W}(s)=-\int_{0}^{\infty} W\left(s^{\prime}\right) \lambda_{z}\left(s-s^{\prime}\right) d s^{\prime}=-\int_{0}^{\infty} S\left(s^{\prime}\right) \lambda_{z}^{\prime}\left(s-s^{\prime}\right) d s^{\prime}
$$

(here the convention is that $\mathcal{W}$ is positive for energy gain). For a Gaussian bunch

$$
\mathcal{W}(s)=-\frac{2 \sqrt{2}}{\sqrt{\pi} \sigma_{z}}\left[e^{-\left(s / \sigma_{z}\right)^{2} / 2} \ln \frac{a}{\sigma_{z}}+f\left(\frac{s}{\sigma_{z}}\right)\right]
$$

with

$$
f(x)=\int_{0}^{\infty}\left(x-x^{\prime}\right) \ln x^{\prime} e^{-\left(x-x^{\prime}\right)^{2} / 2} d x^{\prime}
$$


The function $f(x)$ is shown in Fig. 3. In Fig. 4 we present the bunch wake for three values of $a / \sigma_{z}$. The rms of the bunch wake for a Gaussian is given by

$$
\mathcal{W}_{\text {rms }}=\frac{1}{\sigma_{z}}\left[0.197 \ln ^{2} \frac{a}{\sigma_{z}}+0.264 \ln \frac{a}{\sigma_{z}}+1.302\right]^{1 / 2} .
$$

Finally, note that numerical, time-domain simulations of this problem have been performed by H. Schlarb, and good agreement with Eq. 26 was found [7].

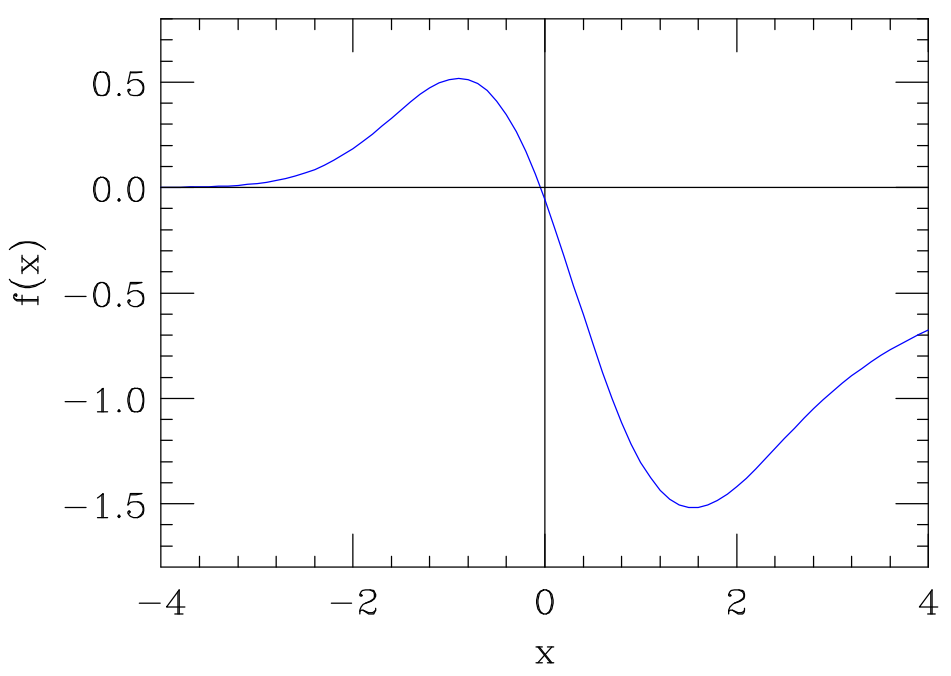

FIG. 3: The function $f(x)$ defined by Eq. 27.

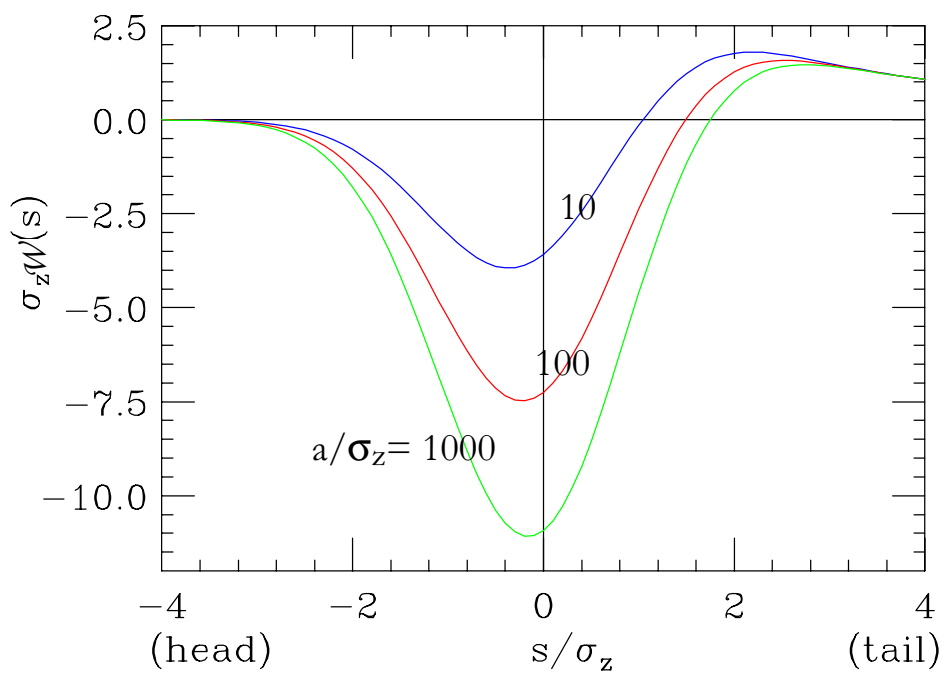

FIG. 4: For Gaussian bunches, the function $\sigma_{z} \mathcal{W}(s)$ for three values of $a / \sigma_{z}$. 


\section{BEAM WITH WIDTH LARGER THAN LENGTH}

\section{A Longitudinal Impedance}

Up to now we have assumed that both driving and test particles are on axis. For the LCLS spoiler problem that we are interested in, however, the beam's transverse size is larger than the longitudinal size. The transverse size will give rise to a transverse impedance, and the transverse size will also cut off both the longitudinal and transverse impedances at high frequencies. Here we sketch the longitudinal calculations (leaving details for Appendix B) and describe the transverse calculations in detail.

Consider now the model sketched in Fig. 5. Again the problem is cylindrically-symmetric, and again there is no hole in the foil. The beam has radial size $\sigma_{r}$ and longitudinal size $\sigma_{z}$, with $\sigma_{z} \ll \sigma_{r} \ll a$. For impedance calculations we consider as driving charge a ring of radius $r_{d}$ (which generates purely monopole fields), and calculate the effect on a test particle following at offset $r$, with $r_{d} \ll a$ and $r \ll \sigma_{r}$. Note that in our geometry with no beam hole, monopole fields generate both a transverse force and a transverse variation in the longitudinal force. Note also that in this case the combination of high frequencies $\left(\omega a / c \sim a / \sigma_{r} \gg 1\right)$ and the condition $r \ll \sigma_{r}$ will allow us to perform wakefield calculations analytically.

The Coulomb source field of the driving ring is now given by

$$
\left(E_{r}\right)_{s}=\frac{2 e N}{r} \delta(z-c t) H\left(r-r_{d}\right)
$$

instead of Eq. 8, and upon field matching the expansion coefficients become

$$
C_{n}=-\frac{4 i e N J_{0}\left(\frac{j_{0 n} r_{d}}{a}\right)}{c k_{z n} j_{0 n}^{2} J_{1}^{2}\left(j_{0 n}\right)}
$$

instead of Eq. 10. The dependence of the longitudinal impedance on the test particle offset $r$ is simply $J_{0}\left(j_{0 n} r / a\right)$ (see Eq. 4), and the real part of the longitudinal impedance becomes:

$$
R\left(r_{d}, \omega\right)=\frac{8}{c} \sum_{n}^{\prime} \frac{J_{0}\left(\frac{j_{0 n} r_{d}}{a}\right) J_{0}\left(\frac{j_{0 n} r}{a}\right)}{\sqrt{1-\left(\frac{j_{0 n} c}{\omega a}\right)^{2}} j_{0 n}^{2} J_{1}^{2}\left(j_{0 n}\right)} .
$$




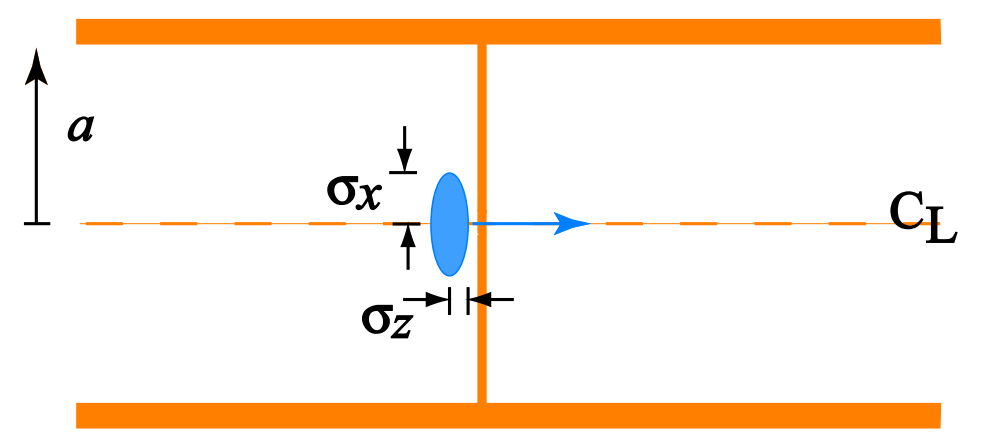

FIG. 5: A sketch of the model for the transverse wake calculation. The beam pipe and beam are both cylindrically symmetric.

In Appendix B we work out details of the longitudinal calculation. In order to obtain an analytical result we calculate a wakefield weighted by a transverse, Gaussian distribution; i.e. we define

$$
\bar{W}(s) \equiv \frac{1}{\sigma_{r}^{2}} \int_{0}^{\infty} W\left(r_{d}, s\right) e^{-\frac{1}{2}\left(r_{d} / \sigma_{r}\right)^{2}} r_{d} d r_{d} .
$$

(Let an over bar designate a function that has been averaged over the driving charge radius, $r_{d}$.) We finally obtain a (transversely averaged) step function response

$$
\bar{S}(s) \approx 4 H(s) \ln \frac{a}{\sigma_{r}} \quad\left[r \ll \sigma_{r}\right]
$$

Comparing with Eq. 24, we note that the beam's transverse size reduces the step function response by the factor $\ln \left(a / \sigma_{r}\right) / \ln (a / s)$. The bunch wake, assuming the longitudinal distribution is also Gaussian, becomes

$$
\overline{\mathcal{W}}(s) \approx-\frac{2 \sqrt{2}}{\sqrt{\pi} \sigma_{z}} e^{-\left(s / \sigma_{z}\right)^{2} / 2} \ln \frac{a}{\sigma_{r}} \quad\left[r, \sigma_{z} \ll \sigma_{r}\right] .
$$

Comparing with Eq. 26 we see that the transverse beam size eliminates the non-symmetric part of the bunch wake, and reduces the symmetric part by the factor $\sim \ln \left(a / \sigma_{r}\right) / \ln \left(a / \sigma_{z}\right)$. Finally, averaging over the longitudinal distribution we find that

$$
\langle\overline{\mathcal{W}}\rangle=\frac{2}{\sqrt{\pi} \sigma_{z}} \ln \frac{a}{\sigma_{r}} \quad\left[r, \sigma_{z} \ll \sigma_{r}\right]
$$

Note that $\overline{\mathcal{W}}(s)$ is independent of $r$ to first order. That is because in the calculation we approximated $J_{0}(x \omega r / c) \approx 1$, where $x$ is a variable $\sim 1$. To estimate the $r$ dependence we can take the next order expansion of the Bessel function: $-\frac{1}{4}(x \omega r / c)^{2} \sim-\frac{1}{4}\left(r / \sigma_{r}\right)^{2}$. 


\section{B Transverse Impedance}

The transverse impedance due to a ring of charge $e N$ and radius $r_{d}$ is

$$
Z_{r}\left(r_{d}, \omega\right)=-\frac{i}{e N} \int_{-\infty}^{\infty}\left[\tilde{E}_{r}\left(r_{d}, r, z ; \omega\right)-\tilde{H}_{\phi}\left(r_{d}, r, z ; \omega\right)\right] e^{-i \omega z / c} d z
$$

Using Eqs. 5,6 we find that the real part of the transverse impedance becomes

$$
R_{r}\left(r_{d}, \omega\right)=-\frac{8}{\omega a} \sum_{n}^{\prime} \frac{J_{0}\left(\frac{j_{0 n} r_{d}}{a}\right) J_{1}\left(\frac{j_{0 n} r}{a}\right)}{\sqrt{1-\left(\frac{j_{0 n} c}{\omega a}\right)^{2}} j_{0 n} J_{1}^{2}\left(j_{0 n}\right)} .
$$

H. Schlarb has shown that, in our geometry, where a beam passes through a foil that is oriented perpendicular to the beam trajectory, the Panofsky-Wenzel theorem [8] still applies [7]. Note that the general form of our transverse impedance, Eq. 37, and that of the longitudinal impedance, Eq. 31, do satisfy this theorem: $R_{r}=(c / \omega) \partial R / \partial r$.

Now let us again assume high frequencies $(\omega a / c \gg 1)$, which will allow us to replace the sum with an integral. Using large $n$ approximations $j_{0 n} \approx n \pi$ and $J_{1}\left(j_{0 n}\right) \approx \sqrt{2 / n}(-1)^{n} / \pi$ we obtain

$$
\begin{aligned}
R_{r}\left(r_{d}, \omega\right) & \approx-\frac{4 \pi}{\omega a} \int_{0}^{\frac{\omega a}{\pi c}} \frac{J_{0}\left(\frac{n \pi r_{d}}{a}\right) J_{1}\left(\frac{n \pi r}{a}\right) d n}{\sqrt{1-\left(\frac{n \pi c}{\omega a}\right)^{2}}} \\
& =-\frac{4}{c} \int_{0}^{1} \frac{J_{0}\left(\frac{x \omega r_{d}}{c}\right) J_{1}\left(\frac{x \omega r}{c}\right) d x}{\sqrt{1-x^{2}}}
\end{aligned}
$$

\section{Transverse Wakefield}

The transverse wakefield is given by

$$
W_{r}\left(r_{d}, s\right)=\frac{2}{\pi} H(s) \int_{0}^{\infty} R_{r}\left(r_{d}, \omega\right) \sin \frac{\omega s}{c} d \omega
$$

Analogously to the longitudinal case we define an averaged transverse wake (averaged over the radial charge distribution), which, for a Gaussian radial distribution, is given by

$$
\bar{W}_{r}(s)=\frac{1}{\sigma_{r}^{2}} \int_{0}^{\infty} W_{r}\left(r_{d}, s\right) e^{-\frac{1}{2}\left(r_{d} / \sigma_{r}\right)^{2}} r_{d} d r_{d}
$$

Combining Eqs. 38-40, and interchanging the order of integration, we obtain

$$
\bar{W}_{r}(s)=-\frac{8}{\pi c \sigma_{r}^{2}} H(s) \int_{0}^{1} \frac{d x}{\sqrt{1-x^{2}}} \int_{0}^{\infty} d \omega J_{1}\left(\frac{x \omega r}{c}\right) \sin \frac{\omega s}{c} \int_{0}^{\infty} r_{d} d r_{d} J_{0}\left(\frac{x \omega r_{d}}{c}\right) e^{-\frac{1}{2}\left(r_{d} / \sigma_{r}\right)^{2}} .
$$


The integration over $r_{d}$ yields

$$
\frac{1}{\sigma_{r}^{2}} \int_{0}^{\infty} r_{d} d r_{d} J_{0}\left(\frac{x \omega r_{d}}{c}\right) e^{-\frac{1}{2}\left(r_{d} / \sigma_{r}\right)^{2}}=e^{-\frac{1}{2}\left(x \sigma_{r} \omega / c\right)^{2}}
$$

In the integral over $\omega$, since the test particle offset is small $\left(r \ll \sigma_{r}\right)$, the Gaussian limits the argument of $J_{1}$ to small values; therefore, we can let $J_{1}\left(\frac{x \omega r}{c}\right) \approx \frac{1}{2}(x \omega r / c)$. We obtain

$$
\frac{2}{\pi} \int_{0}^{\infty} \omega d \omega \sin \frac{\omega s}{c} e^{-\frac{1}{2}\left(x \sigma_{r} \omega / c\right)^{2}}=\sqrt{\frac{2}{\pi}}\left(\frac{s c^{2}}{x^{3} \sigma_{r}^{3}}\right) e^{-\frac{1}{2}\left(\frac{s}{x \sigma_{r}}\right)^{2}} .
$$

The last integral is

$$
\int_{0}^{1} \frac{d x}{x^{2}} \frac{e^{-\frac{1}{2}\left(\frac{s}{x \sigma_{r}}\right)^{2}}}{\sqrt{1-x^{2}}}=\sqrt{\frac{\pi}{2}} e^{-\frac{1}{2}\left(s / \sigma_{r}\right)^{2}} .
$$

Combining the results, we obtain

$$
\bar{W}_{r}(s)=-\frac{2 r}{\sigma_{r}^{2}} H(s) e^{-\frac{1}{2}\left(s / \sigma_{r}\right)^{2}} \quad\left[r \ll \sigma_{r}\right]
$$

Note that the overall minus sign in front of the expression for $\bar{W}_{r}$ implies a focusing wake. Finally, convolving with the longitudinal Gaussian distribution we obtain the transverse bunch wake

$$
\overline{\mathcal{W}}_{r}(s)=-\frac{r}{\sigma_{r}^{2}}\left[1+\operatorname{erf}\left(\frac{s}{\sqrt{2} \sigma_{z}}\right)\right] e^{-\frac{1}{2}\left(s / \sigma_{r}\right)^{2}} \quad\left[r, \sigma_{z} \ll \sigma_{r}\right] .
$$

For the LCLS spoiler problem, we can use this symmetric, wide beam model to estimate the wakefield induced energy loss and transverse kick near the center of the beam. Using Eq. 34, taking $s=0, \sigma_{r}=\sigma_{x}$, and $\sigma_{z}$ as given in Table I, we find that $\overline{\mathcal{W}}=-3 \times 10^{4} / \mathrm{m}$, which corresponds to a relative energy change of $\delta=-6.5 \times 10^{-5}$. Using Eq. 46, taking $r=\Delta x$, we find that $\overline{\mathcal{W}}_{r}=-\Delta x / \sigma_{x}^{2}=-18 / \mathrm{m}$, which corresponds to a kick angle in $x$ of $-0.05 \mu \mathrm{r}$; this is negligible compared to the angular spread in the bunch, $\sigma_{x^{\prime}}=2 \mu \mathrm{rad}$. The rms energy and transverse kick variation over target particles (say, for particles with $|s| \lesssim \sigma_{z s}$ and $\left.|x| \leq \Delta x\right)$ would be a small fraction of these results.

This is a simple model of the LCLS spoiler foil problem. We now consider a more complex one, taking into account the $x-z$ correlation of the beam. 


\section{GREEN FUNCTION}

With the cylindrically symmetric model used so far the effect on target particles in the beam is very small. The real LCLS spoiler problem, however, is not cylindrically symmetric. Even if we keep the approximation that there is no slot in the foil (as we shall continue to do), the symmetry is broken because: the beam has an $x$ - $z$ tilt and the transverse beam sizes are not equal $\left(\sigma_{x} \neq \sigma_{y}\right)$. We can find a Green function, however, that allows us to obtain the wake effect even for beams without cylindrical symmetry.

For a drive particle on-axis and a test particle following at radius $r$ we see from Eq. 37 that the real part of the radial impedance becomes

$$
R_{r}(\omega)=-\frac{8}{\omega a} \sum_{n}^{\prime} \frac{J_{1}\left(\frac{j_{0 n} r}{a}\right)}{\sqrt{1-\left(\frac{j_{0 n} c}{\omega a}\right)^{2}} j_{0 n} J_{1}^{2}\left(j_{0 n}\right)} .
$$

Assuming high frequencies $(\omega a / c \gg 1)$ we can replace the sum by an integral

$$
\begin{aligned}
R_{r}\left(r_{d}, \omega\right) & \approx-\frac{4}{c} \int_{0}^{1} \frac{J_{1}\left(\frac{x \omega r}{c}\right) d x}{\sqrt{1-x^{2}}} \\
& =-\frac{8}{r \omega} \sin ^{2}\left(\frac{r \omega}{2 c}\right) .
\end{aligned}
$$

Performing the sine transform we obtain the radial wake

$$
W_{r}(r, s)=-\frac{4}{r} H(s) H(r-s)
$$

Note that this result can also be obtained by direct integration of the transition radiation fields that are calculated in Ref. [9] for a thin foil in free space. For a Gaussian distributed line charge we convolve Eq. 49 with the charge distribution, and obtain

$$
\mathcal{W}_{r}(r, s)=-\frac{2}{r}\left[\operatorname{erf}\left(\frac{s}{\sqrt{2} \sigma_{z}}\right)-\operatorname{erf}\left(\frac{-r+s}{\sqrt{2} \sigma_{z}}\right)\right] .
$$

A plot of $\mathcal{W}_{r}(r, s)$, for several values of $r / \sigma_{z}$ is given in Fig. 6. Note that for small $r / \sigma_{z}$ the shape of the wake becomes resistive, and for large $r / \sigma_{z}$ capacitive.

Note that $W_{r}$ is independent of $a$. This means that we can consider the limit $a \rightarrow \infty$ corresponding to a foil in free space. Then our assumption that the driving particle is on axis can be dropped, and Eq. 49 is valid also for an off-axis driving particle - in this case $r$ 


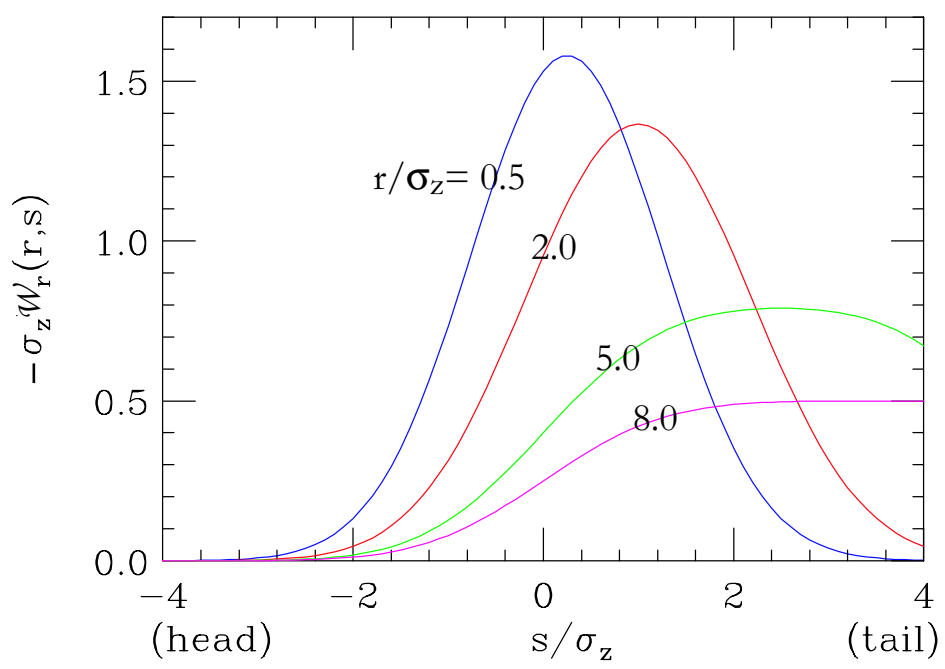

FIG. 6: For Gaussian bunches, the function $-\sigma_{z} \mathcal{W}_{r}(r, s)$ for several values of $r / \sigma_{z}$.

becomes the relative offset between driving and test particles. This function can, therefore, be used as a Green function for the transverse wake of a bunch of any shape, even one that is not cylindrically symmetric. Note, however, that this approximation for Green function neglects higher multipole components of the fields, and therefore is valid only for driving and test particles with off-axis distance small compared to $a$.

\section{A Wakes of a Tilted, Tri-Gaussian Bunch}

Consider now a tri-Gaussian bunch, tilted in the $x-z$ plane by small angle $\alpha$, as is found at the LCLS spoiler, and as sketched in Fig. 1 (but with no foil slot). Let $\alpha>0$ when the bunch head is at $x<0$. Using Eq. 50, the transverse force at test position $(x, y, s)$ is

$$
\begin{aligned}
\overline{\mathcal{W}}_{x}=\frac{1}{(2 \pi)^{3 / 2} \sigma_{z s} \sigma_{x} \sigma_{y}} \iiint d s^{\prime} d x^{\prime} d y^{\prime} e^{-\frac{1}{2}\left[\frac{\left(s^{\prime}-\alpha x^{\prime}\right)^{2}}{\sigma_{z s}^{2}}+\frac{x^{\prime 2}}{\sigma_{x}^{2}}+\frac{y^{\prime 2}}{\sigma_{y}^{2}}\right]} W_{r}\left(\left|\mathbf{r}-\mathbf{r}^{\prime}\right|, s-s^{\prime}\right) \frac{\left(x-x^{\prime}\right)}{\left|\mathbf{r}-\mathbf{r}^{\prime}\right|} \\
=-\frac{1}{\pi \sigma_{x} \sigma_{y}} \iint d x^{\prime} d y^{\prime} \frac{\left(x-x^{\prime}\right)}{\left|\mathbf{r}-\mathbf{r}^{\prime}\right|^{2}} e^{-\frac{1}{2}\left[\frac{x^{\prime 2}}{\sigma_{x}^{2}}+\frac{y^{\prime 2}}{\sigma_{y}^{2}}\right]}\left[\operatorname{erf}\left(\frac{s-\alpha x^{\prime}}{\sqrt{2} \sigma_{z s}}\right)\right. \\
\left.-\operatorname{erf}\left(\frac{-\left|\mathbf{r}-\mathbf{r}^{\prime}\right|+s-\alpha x^{\prime}}{\sqrt{2} \sigma_{z s}}\right)\right]
\end{aligned}
$$

where $\left|\mathbf{r}-\mathbf{r}^{\prime}\right| \equiv \sqrt{\left(x-x^{\prime}\right)^{2}+\left(y-y^{\prime}\right)^{2}}$. (From here on, $\overline{\mathcal{W}}$ will represent the wake of a tilted, tri-Gaussian bunch.)

Let us assume that, as for the LCLS spoiler problem, $\sigma_{x} \gg \sigma_{y}, \sigma_{z} \gg \sigma_{z s}$, and that we are interested in the wake at particle positions with small $x\left(|x| \ll \sigma_{x}\right)$. We can estimate $\overline{\mathcal{W}}_{x}$ 
by analyzing Eq. 51: First note that the error function, erf, of a large positive number is 1, and that erf of a large negative number is -1 . Suppose first that $\alpha>0$. For positive source terms $\left(x^{\prime}>0\right.$ in Eq. 51), the arguments of both erf terms will tend to be large negative numbers, and the total wake effect will be $\sim 0$; for negative source terms $\left(x^{\prime}<0\right)$, the first erf term will tend to be $\sim 1$ while the second erf term will still be $\sim-1$. The asymmetry is a consequence of the fact that the source particle must be in front of the test particle for a non-zero wake effect. Note that source particles over a scale $x^{\prime} \sim \sigma_{x}$ contribute to the transverse wake. And note that if $\alpha<0$ the sign of the force will change. For a test particle near the origin in $x$ (with $|x| \ll \sigma_{x}$ ), Eq. 51 becomes

$$
\begin{aligned}
\overline{\mathcal{W}}_{x} & \approx \frac{4}{\pi \sigma_{x} \sigma_{y}} \operatorname{Sign}(\alpha) \int_{-\infty}^{0} d x^{\prime} \int_{0}^{\infty} d y^{\prime} \frac{x^{\prime}}{x^{\prime 2}+y^{\prime 2}} e^{-\frac{1}{2}\left(\frac{x^{\prime 2}}{\sigma_{x}^{2}}+\frac{y^{\prime 2}}{\sigma_{y}^{2}}\right)} \\
& \approx-\sqrt{\frac{8}{\pi}} \frac{1}{\sigma_{x}} \operatorname{Sign}(\alpha) \ln \left(\frac{2 \sigma_{x}}{\sigma_{y}}\right) .
\end{aligned}
$$

In moving from the first to the second line above we have used the assumption $\sigma_{y} \ll \sigma_{x}$. Note that the kick is in the same direction that the head of the beam points to (downward in the sketch Fig. 1). Eq. 52 is valid for target beam particles when $\sigma_{x} \gg \sigma_{y}, \sigma_{z} \gg \sigma_{z s}$.

For the LCLS beam parameters of Table I, Eq. 52 yields $\overline{\mathcal{W}}_{x}=-2430 / \mathrm{m}$. We also perform numerical calculations - using the general equation, Eq. 51-to find the average and rms force over target particles (i.e. beam particles with $|x|<\Delta x$; about $4 \%$ of the beam). We obtain an average wake $\left\langle\overline{\mathcal{W}}_{x}\right\rangle=-1960 / \mathrm{m}$, which corresponds to an average $x$ kick of $-3.9 \mu \mathrm{rad}$ (compare: $\sigma_{x^{\prime}}=2 \mu \mathrm{rad}$ ). An average kick, however, can be compensated for; rather, it is the variation in kick, over the target particles, which is important. We find numerically that the rms deviation wake $\left(\overline{\mathcal{W}}_{x}\right)_{r m s}=165 / \mathrm{m}$, corresponding to an rms kick angle of $0.3 \mu \mathrm{rad}$, which is small.

To obtain the vertical wake $\overline{\mathcal{W}}_{y}$, we need only replace the factor $\left(x-x^{\prime}\right) /\left|\mathbf{r}-\mathbf{r}^{\prime}\right|$ in the integrals of Eq. 51 by $\left(y-y^{\prime}\right) /\left|\mathbf{r}-\mathbf{r}^{\prime}\right|$. For the target particles, we see from symmetry that $\left\langle\overline{\mathcal{W}}_{y}\right\rangle=0$; and numerically we obtain an rms of $\left(\overline{\mathcal{W}}_{y}\right)_{r m s}=540 / \mathrm{m}$, corresponding to an rms kick angle of $1.1 \mu \mathrm{rad}$. Note that the deviation is not small compared to $\sigma_{y^{\prime}}=2 \mu \mathrm{rad}$. In Fig. 7 we plot $\overline{\mathcal{W}}_{y}$ vs. $y$, for $x=-\Delta x, 0, \Delta x$. Note the linear dependence on $y$ near the origin. This suggests that a vertically focusing quadrupole may be used to correct much of 
the wakefield effect. Performing a simple, linear optimization, we find that indeed this is true. With a quad with a $95 \mathrm{~m}$ focal length, the deviation in vertical angular spread can be reduced to $0.3 \mu \mathrm{rad}$, which is now small compared to the natural beam divergence.

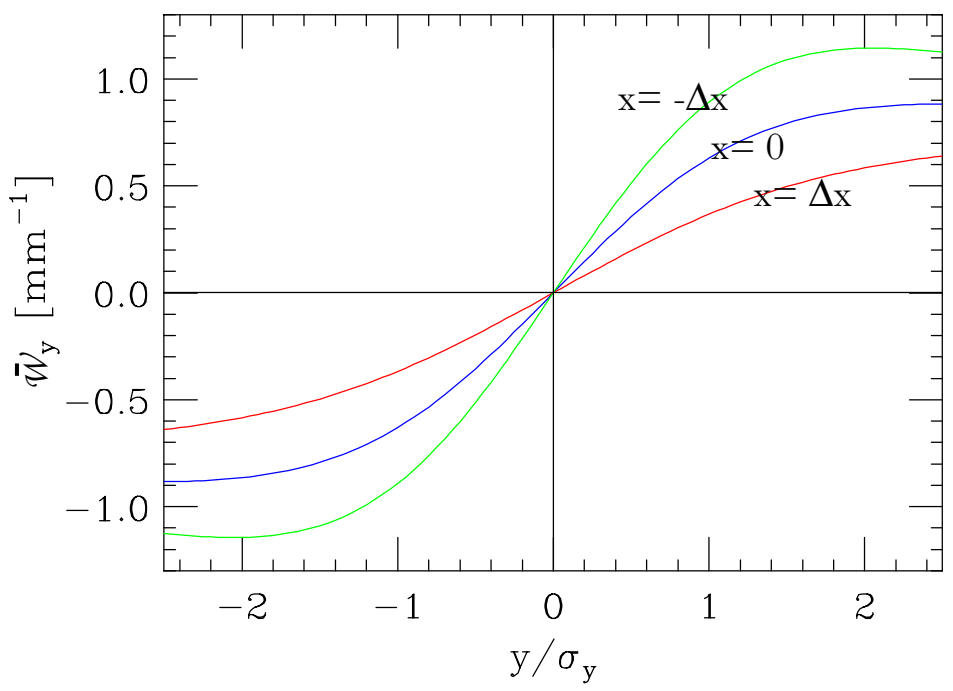

FIG. 7: For LCLS spoiler parameters: $\overline{\mathcal{W}}_{y}$ vs. $y$, for $x=-\Delta x, 0, \Delta x$.

To find the longitudinal wakefield of the tilted beam we use the Panofsky-Wenzel Theorem. In a beam pipe of radius $a$ the longitudinal wake can be obtained from the horizontal wake by the relation

$$
\overline{\mathcal{W}}_{z}(x, y, s)=\int_{x}^{\sqrt{a^{2}-y^{2}}} \frac{\partial \overline{\mathcal{W}}_{x}\left(x_{1}, y, s\right)}{\partial s} d x_{1}
$$

The upper limit of integration is chosen so that $\overline{\mathcal{W}}_{z}$ on the beam pipe wall is zero. Using Eq. 51, we obtain $\partial \overline{\mathcal{W}}_{x} / \partial s$ :

$$
\begin{aligned}
\frac{\partial \overline{\mathcal{W}}_{x}}{\partial s}=-\frac{\sqrt{2}}{\pi^{3 / 2} \sigma_{x} \sigma_{y} \sigma_{z s}} \iint & d x^{\prime} d y^{\prime} \frac{\left(x-x^{\prime}\right)}{\left|\mathbf{r}-\mathbf{r}^{\prime}\right|^{2}} e^{-\frac{1}{2}\left(\frac{x^{\prime 2}}{\sigma_{x}^{2}}+\frac{y^{\prime 2}}{\sigma_{y}^{2}}\right)} \times \\
& \times\left[e^{-\frac{1}{2}\left(s-\alpha x^{\prime}\right)^{2} / \sigma_{z s}^{2}}-e^{-\frac{1}{2}\left(-\left|\mathbf{r}-\mathbf{r}^{\prime}\right|+s-\alpha x^{\prime}\right)^{2} / \sigma_{z s}^{2}}\right] .
\end{aligned}
$$

Eqs. 53,54, give the general solution for $\overline{\mathcal{W}}_{z}$. We see that there are 2 contributions. Let us demonstrate that, for the LCLS spoiler problem, the second contribution is much smaller than the first, and can be dropped. First, note that the integrand is well behaved and has no singularities. For the first contribution, note that the last exponential is significant only for source particles with $x^{\prime} \lesssim \sigma_{z s} / \alpha=\sigma_{z s} \sigma_{x} / \sigma_{z}$. For the second contribution, however, 
the last exponential is significant only for $\left|\mathbf{r}-\mathbf{r}^{\prime}\right| \lesssim \sigma_{z s}$, which involves a much smaller fraction of beam source particles. The relative number of significant source particles here compared to the first term is $\sigma_{z s} \sigma_{z} /\left(\sigma_{x} \sigma_{y}\right)=8 \times 10^{-4}$; therefore, this contribution is very small and this term can be dropped. For what remains, to get a scale of the important distances involved note that only those source particles with $\left|x-x^{\prime}\right| \lesssim \sigma_{z s} / \alpha=50 \mu \mathrm{m}$ and $\left|y-y^{\prime}\right| \lesssim \sigma_{y}=100 \mu \mathrm{m}$ contribute significantly to the wake.

Inserting Eq. 54, without the second term, into Eq. 53, we obtain

$$
\begin{aligned}
\overline{\mathcal{W}}_{z}=-\frac{1}{\sqrt{2} \pi^{3 / 2} \sigma_{x} \sigma_{y} \sigma_{z s}} & \iint d x^{\prime} d y^{\prime} \ln \left[\frac{\left(\sqrt{a^{2}-y^{2}}-x^{\prime}\right)^{2}+\left(y-y^{\prime}\right)^{2}}{\left|\mathbf{r}-\mathbf{r}^{\prime}\right|^{2}}\right] \times \\
& \times e^{-\frac{1}{2}\left(\frac{x^{\prime 2}}{\sigma_{x}^{2}}+\frac{y^{\prime 2}}{\sigma_{y}^{2}}\right)} e^{-\frac{1}{2}\left(s-\alpha x^{\prime}\right)^{2} / \sigma_{z s}^{2}} .
\end{aligned}
$$

In Eq. 55, for LCLS type parameters, the final Gaussian is much narrower than the other $x^{\prime}$ Gaussian, so it behaves like a delta function. It can be approximated as $e^{-\frac{1}{2}\left(s-\alpha x^{\prime}\right)^{2} / \sigma_{z s}^{2}} \approx$ $\sqrt{2 \pi} \sigma_{z s} \delta\left(s-\alpha x^{\prime}\right)$. Note also that the numerator in the $\log$ term can be replaced by $a^{2}$. We then obtain (for $|x| \ll \sigma_{x}$ )

$$
\begin{aligned}
\overline{\mathcal{W}}_{z} & \approx-\frac{1}{\pi \sigma_{z} \sigma_{y}} \int_{-\infty}^{\infty} d y^{\prime} \ln \left[\frac{a^{2}}{x^{2}+\left(y-y^{\prime}\right)^{2}}\right] e^{-\frac{1}{2} \frac{y^{\prime 2}}{\sigma_{y}^{2}}} \\
& \approx-\sqrt{\frac{8}{\pi}} \frac{1}{\sigma_{z}} \ln \left(\frac{\sqrt{2} a}{\sigma_{y}}\right) .
\end{aligned}
$$

Eq. 56 is valid for target beam particles when $a \gg \sigma_{x} \gg \sigma_{y}, \sigma_{z} \gg \sigma_{z s}$.

For the LCLS beam parameters of Table I, Eq. 56 yields $\overline{\mathcal{W}}_{z}=-9.0 \times 10^{4} / \mathrm{m}$. Numerically solving Eq. 55 for the target particles, we obtain an average wake $\left\langle\overline{\mathcal{W}}_{z}\right\rangle=-8.2 \times 10^{4} / \mathrm{m}$, equivalent to an average relative energy change $\langle\delta\rangle=-1.6 \times 10^{-4}$; and an rms wake $\left(\overline{\mathcal{W}}_{z}\right)_{r m s}=4300 / \mathrm{m}$, equivalent to $(\delta)_{r m s}=9 \times 10^{-6}$. Note that the rms deviation is small compared to the intrinsic relative energy spread $\left(\sigma_{\delta}\right)_{\text {in }}=6 \times 10^{-5}$. A summary of our analytical and numerical results for LCLS parameters is given in Table II.

Our derivations are based on the assumption of perfectly conducting beam pipe walls and foil. We can now estimate the validity of this assumption for the LCLS parameters. It was pointed out above that the main contribution to the integral of the transverse wake comes from distances $x \sim \sigma_{x}$, and to the integral of the longitudinal wake from distances 
TABLE II: For the LCLS spoiler foil: summary of wake effects in the three directions on target beam particles. The average horizontal force points in the same direction as the head of the beam.

\begin{tabular}{lcccc}
\hline \multirow{2}{*}{ Direction } & \multicolumn{2}{c}{ Average } & Rms & Units \\
& Analytical & Numerical & Numerical & \\
\hline Horizontal $\left(x^{\prime}\right)$ & 4.8 & 3.9 & 0.3 & $\mu \mathrm{rad}$ \\
Vertical $\left(y^{\prime}\right)$ & 0 & 0 & 1.1 & $\mu \mathrm{rad}$ \\
Longitudinal $(\delta)$ & -18. & -16. & 0.9 & $10^{-5}$ \\
\hline \hline
\end{tabular}

$x \sim \sigma_{s z} / \alpha$. This means that characteristic frequencies $\omega$ that are responsible for the wakes are $c / \sigma_{x}=10^{11} \mathrm{~s}^{-1}$ and $c \alpha / \sigma_{z s}=6 \times 10^{12} \mathrm{~s}^{-1}$, respectively. At these frequencies the conductivity of metals such as copper is still large [10] and the perfect conductivity model is a good approximation.

\section{CONCLUSION}

We have obtained longitudinal and transverse impedances and wakefields for several models of the proposed LCLS spoiler foil using field matching. The environment in all models is a cylindrically symmetric, perfectly conducting beam pipe which, at one longitudinal position, is filled by a thin, metallic foil. A high energy beam passes through the pipe and through the foil. For the first model we consider a pencil beam; for the second, a cylindrically symmetric, bi-Gaussian beam that is wider than it is long. Finally, we consider a more realistic model, an asymmetric beam with $x-z$ tilt.

For the LCLS we have studied the effect of the wakefields generated at the foil on target beam particles, i.e. the small fraction of beam that is meant to pass through a slot in the foil. We have used the Green function of our model to generate wakes of a tilted, triGaussian bunch as will be found at the LCLS spoiler. We have used these results to obtain approximate formulas for the average wakefields experienced by target particles within the bunch, and have also obtained numerically the average and rms wakefield effects in the three directions. The average effects agree well with the analytical formulas, and the rms 
deviations appear to be within tolerances for the LCLS. The kick closest to tolerances may be the rms vertical kick, though it appears that a simple, vertical focusing quad will be able to reduce this to negligible levels also.

This has been a first attempt to understand the wakefields at the LCLS spoiler foil. An important approximation in all our calculations is the absence of slot or hole for target particles to pass through. We believe that the asymmetry in the beam combined with the small size (compared to the beam size) of the slot mean that the presence of such a slot is not a large perturbation to the problem, though more work is needed to study this question in detail.

\section{ACKNOWLEDGMENTS}

The authors thank A. Chao, M. Cornacchia, P. Emma, and Z. Huang for helpful discussions of this problem, and H. Schlarb for describing his numerical time-domain results and carefully reading the manuscript. This work was supported by the Department of Energy, contract DE-AC03-76SF00515.

\section{APPENDIX A}

We want to find the sum

$$
S_{2}=\sum_{n}^{\prime}\left[\frac{1}{j_{0 n}^{2} J_{1}^{2}\left(j_{0 n}\right)}-\frac{1}{2 n}\right]
$$

with $J_{m}$ the Bessel function of order $m$, and $j_{0 n}$ the $n^{\text {th }}$ zero of $J_{0}(x)$. First note that $\sum_{n}^{\prime}$ indicates that summation is over a large number of terms; we can move the upper limit to $n=\infty$, which will not change the result. To perform this sum we use the relation[11]

$$
\sum_{1}^{\infty} \frac{J_{0}\left(j_{0 n} x\right)}{j_{0 n}^{2} J_{1}^{2}\left(j_{0 n}\right)}=-\frac{1}{2} \ln x
$$

This equation can be rewritten as

$$
\sum_{1}^{A}\left(\frac{J_{0}\left(j_{0 n} x\right)}{j_{0 n}^{2} J_{1}^{2}\left(j_{0 n}\right)}-\frac{1}{2 n}\right)+\sum_{A}^{\infty} \frac{J_{0}\left(j_{0 n} x\right)}{j_{0 n}^{2} J_{1}^{2}\left(j_{0 n}\right)}+\sum_{1}^{A} \frac{1}{2 n}=-\frac{1}{2} \ln x
$$


where $A$ is a large, fixed integer. Let us consider $x$ small enough so that $J_{0}\left(j_{0 n} x\right) \approx 1$ in the first summation on the left hand side of Eq. 59 (i.e. so that $\pi x A \ll 1$ ), and that this term will equal $S_{2}$, the sum that we desire.

For the second term we use large $n$ approximations for $j_{0 n} \approx n \pi$ and $J_{1}\left(j_{0 n}\right) \approx$ $\sqrt{2 / n}(-1)^{n} / \pi$, and replace the sum with an integral; i.e.

$$
\begin{aligned}
\sum_{A}^{\infty} \frac{J_{0}\left(j_{0 n} x\right)}{j_{0 n}^{2} J_{1}^{2}\left(j_{0 n}\right)} & =\frac{1}{2} \int_{\pi x A}^{\infty} \frac{J_{0}(y)}{y} d y \\
& =\left.\frac{1}{2} J_{0}(y) \ln y\right|_{\pi x A} ^{\infty}+\frac{1}{2} \int_{0}^{\infty} J_{1}(y) \ln y d y \\
& =-\frac{1}{2}\left(\ln \pi x A+\gamma_{E}-\ln 2\right)
\end{aligned}
$$

Note that in going from the first to the second line we have used integration by parts, and that the lower limit of the integral in the second line has been set to 0 . The third sum on the left of Eq. 59

$$
\sum_{1}^{A} \frac{1}{2 n}=\frac{1}{2}\left(\gamma_{E}+\ln A\right) .
$$

Finally, combining all the pieces, we note that the $\ln x$ and $\ln A$ terms cancel, and we obtain

$$
S_{2}=\sum_{n}^{\prime}\left[\frac{1}{j_{0 n}^{2} J_{1}^{2}\left(j_{0 n}\right)}-\frac{1}{2 n}\right]=\frac{1}{2} \ln \frac{\pi}{2} .
$$

\section{APPENDIX B}

We want to find the longitudinal wakefield effect for a bunch that is radially Gaussian, with rms size $\sigma_{r}$, and also longitudinally Gaussian, with $\mathrm{rms}$ size $\sigma_{z}$, where $\sigma_{z} \ll \sigma_{r} \ll a$. We are interested in high frequencies, since $\omega a / c \sim a / \sigma_{r} \gg 1$, and small test particle radial position $\left(r \ll \sigma_{r}\right)$.

The general form of the real part of the longitudinal impedance is

$$
R\left(r_{d}, \omega\right)=\frac{8}{c} \sum_{n}^{\prime} \frac{J_{0}\left(\frac{j_{0 n} r_{d}}{a}\right) J_{0}\left(\frac{j_{0 n} r}{a}\right)}{\sqrt{1-\left(\frac{j_{0 n} c}{\omega a}\right)^{2}} j_{0 n}^{2} J_{1}^{2}\left(j_{0 n}\right)} .
$$

The longitudinal wakefield is given by

$$
W\left(r_{d}, s\right)=\frac{2}{\pi} H(s) \int_{0}^{\infty} R_{r}\left(r_{d}, \omega\right) \cos \frac{\omega s}{c} d \omega
$$


The averaged longitudinal wake (averaged over the radial charge distribution), for a Gaussian radial distribution, is given by

$$
\bar{W}(s)=\frac{1}{\sigma_{r}^{2}} \int_{0}^{\infty} W\left(r_{d}, s\right) e^{-\frac{1}{2}\left(r_{d} / \sigma_{r}\right)^{2}} r_{d} d r_{d} .
$$

Combining Eqs. 63-65, and interchanging the order of integration, we obtain

$$
\bar{W}(s)=\frac{16 H(s)}{\pi c \sigma_{r}^{2}} \int_{0}^{\infty} d \omega \cos \frac{\omega s}{c} \int_{0}^{\infty} r_{d} d r_{d} e^{-\frac{1}{2}\left(r_{d} / \sigma_{r}\right)^{2}} \sum_{n}^{\prime} \frac{J_{0}\left(\frac{j_{0 n} r_{d}}{a}\right) J_{0}\left(\frac{j_{0 n} r}{a}\right)}{\sqrt{1-\left(\frac{j_{0 n} c}{\omega a}\right)^{2}} j_{0 n}^{2} J_{1}^{2}\left(j_{0 n}\right)} .
$$

The sum can be rewritten in three pieces $S=S_{1}+S_{2}+S_{3}$ as (remember $\sum_{n}^{\prime}$ means to sum all $n$ up to $n_{\max }$, where $n_{\max }$ is the largest integer $n$ for which $\left.j_{0 n} c /(\omega a) \leq 1\right)$ :

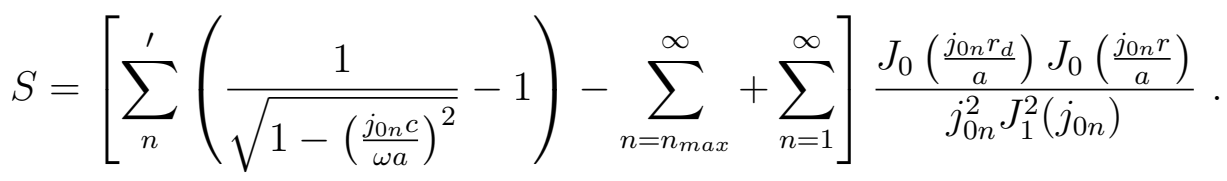

Let us first consider $S_{1}$ and its contribution to $\bar{W}(s)$ of Eq. 66 , that we denote as $\bar{W}_{1}(s)$. For large $\omega a / c, S_{1}$ is dominant at large $n$. Therefore, we can replace the sum $S_{1}$ with an integral. Using large $n$ approximations $j_{0 n} \approx n \pi$ and $J_{1}\left(j_{0 n}\right) \approx \sqrt{2 / n}(-1)^{n} / \pi$ we obtain

$$
S_{1}=\frac{1}{2} \int_{0}^{1} \frac{d x}{x}\left(\frac{1}{\sqrt{1-x^{2}}}-1\right) J_{0}\left(\frac{x \omega r_{d}}{c}\right) J_{0}\left(\frac{x \omega r}{c}\right) .
$$

If we now change the order of integration in Eq. 66, so that we perform the integration over $r_{d}$ first, we obtain

$$
\frac{1}{\sigma_{r}^{2}} \int_{0}^{\infty} r_{d} d r_{d} J_{0}\left(\frac{x \omega r_{d}}{c}\right) e^{-\frac{1}{2}\left(r_{d} / \sigma_{r}\right)^{2}}=e^{-\frac{1}{2}\left(x \sigma_{r} \omega / c\right)^{2}}
$$

Considering next the integration over $\omega$ we see that, since $r \ll \sigma_{r}$, the Gaussian allows us to replace $J_{0}(x \omega r / c)$ by 1 :

$$
\frac{2}{\pi} \int_{0}^{\infty} d \omega \cos \frac{\omega s}{c} e^{-\frac{1}{2}\left(x \sigma_{r} \omega / c\right)^{2}}=\sqrt{\frac{2}{\pi}}\left(\frac{c}{x \sigma_{r}}\right) e^{-\frac{1}{2}\left(\frac{s}{x \sigma_{r}}\right)^{2}} .
$$

The final integration is

$$
\int_{0}^{1} \frac{d x}{x^{2}}\left(\frac{1}{\sqrt{1-x^{2}}}-1\right) e^{-\frac{1}{2}\left(\frac{s}{x \sigma_{r}}\right)^{2}}=\sqrt{\frac{\pi}{2}}\left(\frac{\sigma_{r}}{s}\right)\left[-1+e^{-\frac{1}{2}\left(s / \sigma_{r}\right)^{2}}+\operatorname{erf}\left(\frac{s}{\sqrt{2} \sigma_{r}}\right)\right] .
$$

We finally obtain for the first piece of the (transversely averaged) wake

$$
\bar{W}_{1}(s)=\frac{4}{s} H(s)\left[-1+e^{-\frac{1}{2}\left(s / \sigma_{r}\right)^{2}}+\operatorname{erf}\left(\frac{s}{\sqrt{2} \sigma_{r}}\right)\right] .
$$


For the second part of the wake $\bar{W}_{2}(s)$, which depends on the second sum $S_{2}$, the integrals over $r_{d}$ and $\omega$ are the same. The $x$ integral, however, is

$$
\int_{0}^{1} \frac{d x}{x^{2}} e^{-\frac{1}{2}\left(\frac{s}{x \sigma_{r}}\right)^{2}}=\sqrt{\frac{\pi}{2}}\left(\frac{\sigma_{r}}{s}\right) \operatorname{erf}\left(\frac{s}{\sqrt{2} \sigma_{r}}\right)
$$

leading to

$$
\bar{W}_{2}(s)=-\frac{4}{s} H(s) \operatorname{erf}\left(\frac{s}{\sqrt{2} \sigma_{r}}\right) .
$$

The third part of the wake $\bar{W}_{3}(s)$, depends on the third sum

$$
S_{3}=\sum_{n=1}^{\infty} \frac{J_{0}\left(\frac{j_{0 n} r_{d}}{a}\right) J_{0}\left(\frac{j_{0 n} r}{a}\right)}{j_{0 n}^{2} J_{1}^{2}\left(j_{0 n}\right)} .
$$

For $r \ll r_{d}$ we let $J_{0}\left(j_{0 n} r / a\right) \approx 1$ in the sum, and use [11]

$$
\sum_{n=1}^{\infty} \frac{J_{0}\left(j_{0 n} x\right)}{j_{0 n}^{2} J_{1}^{2}\left(j_{0 n}\right)}=-\frac{1}{2} \ln x .
$$

Since there is no $\omega$ dependence, the integral over $\omega$ becomes simply

$$
\frac{2}{\pi} \int_{0}^{\infty} d \omega \cos \frac{\omega s}{c}=\delta(s)
$$

The integral over $r_{d}$ is

$$
\int_{0}^{\infty} r_{d} d r_{d} \ln \frac{a}{r_{d}} e^{-\frac{1}{2}\left(r / r_{d}\right)^{2}}=\left[\frac{1}{2}\left(\gamma_{E}-\ln 2\right)+\ln \frac{a}{\sigma_{r}}\right]
$$

Thus the third, final part of the wake becomes

$$
\bar{W}_{3}(s)=4 \delta(s)\left[\frac{1}{2}\left(\gamma_{E}-\ln 2\right)+\ln \frac{a}{\sigma_{r}}\right] \approx 4 \delta(s) \ln \frac{a}{\sigma_{r}} .
$$

Finally, combining all three parts, we obtain the total (transversely averaged) longitudinal wake

$$
\begin{aligned}
\bar{W}(s) & =\frac{4}{s} H(s)\left(-1+e^{-\frac{1}{2}\left(s / \sigma_{r}\right)^{2}}\right)+4 \delta(s) \ln \frac{a}{\sigma_{r}} & \\
& \approx 4 \delta(s) \ln \frac{a}{\sigma_{r}} & {\left[r, s \ll \sigma_{r}\right] . }
\end{aligned}
$$




\section{REFERENCES}

[1] P. Emma et al., Sub-femtosecond x-ray pulse from a sase fel using a slotted foil in a bunch compressor chicane, to be presented at the 2003 FEL conference, Tsukuba, Ibaraki, Japan, Sep. 8-12, 2003.

[2] M. Cornacchia et al., The ultimate possibilities of the lcls (2003), to be published.

[3] LCLS Design Study Group, Linac Coherent Light Source (LCLS) Design Study Report, SLAC-R 593, SLAC (2002), /http://www-ssrl.slac.stanford.edu/lcls/CDR.

[4] G. Stupakov, in Joint US-CERN-JAPAN-RUSSIA Accelerator School (2002).

[5] R. Carr and H. Wiedemann, in Handbook of Accelerator Physics and Engineering, edited by A. W. Chao and M. Tigner (World Scientific, 1999), p. 191.

[6] A. Chao and P. Morton, Differential energy loss for a particle in a square pulse of charge traveling between infinitely conducting plates, PEP 0119, SLAC (1975).

[7] H. Schlarb, Numerical simulation of coherent transition radiation excited by a thin metal foil, to be published.

[8] W.K.H. Panofsky and W.A. Wenzel, Review of Scientific Instruments 27, 967 (1956).

[9] A. W. Chao, Physics of collective beam instabilities in high energy accelerators (John Wiley \& Sons, New York, NY, 1993), pp. 97-105.

[10] K. Bane and M. Sands, in Proceedings of Micro Bunches Workshop, Upton, NY, 1995 (American Institute Physics, 1995), p. 131.

[11] A.P. Prudnikov, Y.A. Brychkov, O.I. Marichev, Integrals and Sums-Special Functions (Nauka, Moscow, 1983), p. 690. 